\title{
Chondrocyte-associated antigen and matrix components in a 2- and 3-dimensional culture of rat chondrocytes
}

\author{
ANNA OSIECKA-IWAN, ANNA HYC, JUSTYNA NIDERLA-BIELINSKA and STANISLAW MOSKALEWSKI
}

Department of Histology and Embryology, Medical University of Warsaw, Chalubinskiego 5, PL02-004 Warsaw, Poland

Received June 24, 2008; Accepted September 1, 2008

DOI: $10.3892 / \mathrm{mmr}+00000045$

\begin{abstract}
The goal of this study was to compare expression of chondrocyte-associated antigen (CAA) and cartilage matrix molecules in 2-D (monolayer) and 3-D (Matrigel) culture. Chondrocytes isolated from the cartilage of 3-day-old rats were expanded in monolayer culture for 28 days. CAA expression gradually decreased and was not detected beyond the 96th hour of monolayer culture. Collagen type II and aggrecan mRNA levels decreased during culture. The collagen type I mRNA level increased during the first week and remained high. The increase in the versican mRNA level was less pronounced during the first week and declined slightly after further cultivation. Freshly isolated chondrocytes introduced into Matrigel still expressed CAA after 7 days. Moreover, CAA expression returned in chondrocytes re-cultured in Matrigel after 7 days in monolayer. Similarly, the increase in the mRNA levels of collagen type II and aggrecan in Matrigel was limited to freshly isolated chondrocytes and to those that remained in monolayer for 1 week. Collagen type I mRNA in monolayer and Matrigel cultures of freshly isolated chondrocytes was at a similar level. The introduction of freshly isolated or 7-day monolayer-cultured chondrocytes into Matrigel caused a decrease in the versican mRNA level in comparison with 7- and 14-day 2-D cultures, respectively. On the other hand, chondrocytes seeded in Matrigel after 14 days of monolayer culture did not express CAA, showed decreased levels of collagen type II and aggrecan mRNA and an increase in versican mRNA. In conclusion, it appears that changes in the expression of CAA in 2- and 3-D cultures occur in parallel to changes in typical cartilage matrix molecule expression.
\end{abstract}

\section{Introduction}

Chondrocytes liberated from cartilage matrix and placed in monolayer culture undergo a transition from a polygonal to a

Correspondence to: Dr Anna Osiecka-Iwan, Department of Histology and Embryology, Medical University of Warsaw, Chalubinskiego 5, PL02-004 Warsaw, Poland

E-mail: aosiecka@ib.amwaw.edu.pl

Key words: chondrocyte-associated antigen, chondrogenic expression, two-dimensional culture, Matrigel three-dimensional culture fibroblast-like shape, and change the profile of the macromolecules produced by suppressing collagen type II and proteoglycan synthesis and by the concomitant expression of collagen type I (1-4). These changes can be reverted by transferring chondrocytes from monolayer to 3-D culture in agarose gel (5) or alginate beads (6).

Immunophenotypic analysis of human articular chondrocytes grown in monolayer culture disclosed differential expression of various surface molecules with characteristic upregulation of markers regarded as distinctive for mesenchymal stem cells $(7,8)$. Moreover, isolated chondrocytes bear cartilage matrix, chondrocyte-specific and histocompatibility antigens (9). We recently observed that transplants of rat chondrocytes into rabbits evoked the formation of cytotoxic antibodies that reacted with chondrocytes but not with thymocytes, fibroblasts or endotheliocytes (10). Relating this chondrocyte-associated antigen (CAA) to previously recognized chondrocyte markers requires further study. However, the goal of this study was to establish how CAA phenotypic expression is affected by 2and 3-D cultures of chondrocytes, and to compare its expression with that of typical matrix components produced by chondrocytes or fibroblasts. As a substrate for 3-D culture, we used Matrigel. This medium contains numerous growth factors and has been found to favor expression of collagen type II and aggrecan mRNA in cultures of mesenchymal stem cells from bone marrow (11).

\section{Materials and methods}

Isolation of chondrocytes. Three-day-old inbred Lewis rats served as cartilage donors. Chondrocytes were isolated from the articular-epiphyseal cartilage complexes forming the proximal and distal end of the humerus and head of the femur. Cartilage fragments were first exposed to $0.25 \%$ trypsin in PBS for $45 \mathrm{~min}$ at $37^{\circ} \mathrm{C}$ to remove cells adhering to its surface close to the area of separation from the bone shaft. Subsequently, they were digested with constant stirring in enzymic solution containing $0.25 \%$ collagenase (type I),

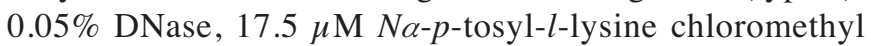
ketone (TLCK) and 1\% antibiotic-antimycotic solution (all from Sigma-Aldrich Chemie, Diesenhofen, Germany) in F-12 medium (Gibco BRL, Paisley, Scotland, UK) for $\sim 3 \mathrm{~h}$ at $37^{\circ} \mathrm{C}$. Following isolation, chondrocytes were filtered through a $40-\mu \mathrm{m}$ mesh nylon filter and rinsed three times with a culture medium composed of F-12 medium supplemented with 10\% FCS (Gibco) and antibiotics (12). 
Monolayer culture of chondrocytes (2-D culture). Chondrocytes were seeded into 35-mm dishes (Corning Inc., Corning, NY, USA) at a density of $5 \times 10^{5}$ cells per dish in $2 \mathrm{ml}$ of culture medium. After the cells reached subconfluency, they were detached with $0.25 \%$ trypsin-EDTA (Sigma), counted in the Bürker chamber and seeded into new vessels at the same density. Passages were carried out after 4, 7, 10, 12, 14, 17, 19, 21 and 24 days (9 population doublings). The cells from the last passage ceased to proliferate when left in culture for an additional 2 or 4 days. Subconfluent cultures were used for isolation of RNA.

Since preliminary observations indicated that chondrocyte surface antigen is removed by trypsin, in another set of experiments chondrocytes were detached from dishes by collagenase. This increased the time needed for the detachment of the cells to $3 \mathrm{~h}$, and was associated with a considerable loss of cells. Chondrocytes, either freshly isolated or cultured for $24,48,60,72,96,148 \mathrm{~h}$ and 7, 14 and 21 days, were used for antigen isolation or for transfer into 3-D culture.

Three-dimensional culture of chondrocytes (3-D culture). Chondrocytes, freshly isolated or cultured for 7 or 14 days in monolayer, were seeded into 24-well plates (Corning) at a density of $5 \times 10^{6}$ cells per $\mathrm{ml}$ of Matrigel (BD Biosciences, Belgium) diluted 1:1 with F-12 medium. BD Matrigel Basement Membrane Matrix, extracted according to the manufacturer's specification from the Engelbrech-HolmSwarm mouse sarcoma, contained (on average) EGF $0.7 \mathrm{ng}$ / $\mathrm{ml}, \mathrm{bFGF}<0.1-0.2 \mathrm{pg} / \mathrm{ml}$, NGF $<0.2 \mathrm{ng} / \mathrm{ml}$, PDGF $12 \mathrm{pg} / \mathrm{ml}$, IGF-1 $16 \mathrm{ng} / \mathrm{ml}$, TGFß $2.3 \mathrm{ng} / \mathrm{ml}$, laminin 56\%, collagen IV $31 \%$ and entactin $8 \%$ in Dulbecco's modified Eagle's medium with $50 \mu \mathrm{g} / \mathrm{ml}$ gentamycin. Each well contained $0.2 \mathrm{ml}$ of cell suspension. The bottom of the wells was pre-covered with Matrigel to prevent the cells from attaching to the plastic. Cultures in Matrigel lasted for 7 days. Matrigel was digested with collagenase and rinsed 3 times with PBS. The liberated cells were used for RNA or CAA isolation.

Chondrocyte-associated antigen preparation. Expression of CAA in 2- or 3-D chondrocyte cultures was detected by Western blot analysis. Chondrocyte surface proteins were extracted with $50 \mathrm{mM}$ Bis-Tris/HCL (pH 7.0) buffer containing $750 \mathrm{mM}$ aminocaproic acid and $125 \mu \mathrm{l}$ of $10 \%$ laurylmaltoside per $1 \mathrm{ml}$ of buffer (all reagents from SigmaAldrich) (13), desalted on PD-10 columns (Amersham Biosciences, Uppsala, Sweden), lyophilized and dissolved in $100 \mu 1$ of distilled water.

Protein determination. One microliter of chondrocyte extract or extraction buffer (blank test) and $9 \mu 1$ deionized water were placed in a flat-bottomed 96-well plate, and $0.2 \mathrm{ml}$ of BCA protein assay reagent (Pierce, Rockford, IL, USA) was added to each well. The plate was incubated at $37^{\circ} \mathrm{C}$ for $30 \mathrm{~min}$. Protein concentration was determined spectrophotometrically at $550 \mathrm{~nm}$ (SLT Spectra Lab Instruments, Crailsheim, Germany).

Electrophoresis and Western blotting. Chondrocyte extracts (10 $\mu \mathrm{g}$ of protein) mixed in sample buffer with or without 2-mercaptoethanol were separated by SDS-polyacrylamide gel

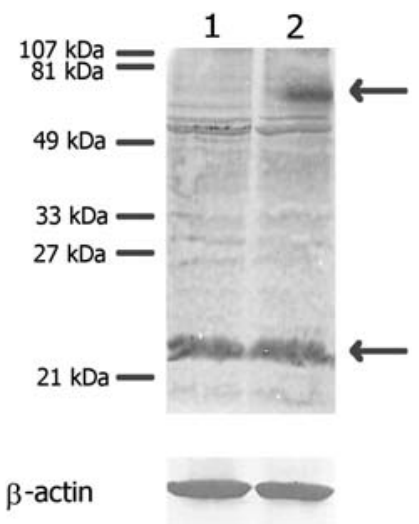

Figure 1. SDS-PAGE and an immunoblot of chondrocyte extracts. Extract in lane 1 was run under a reducing condition; extract in lane 2 was run without 2-mercaptoethanol. Arrows show specific reaction.

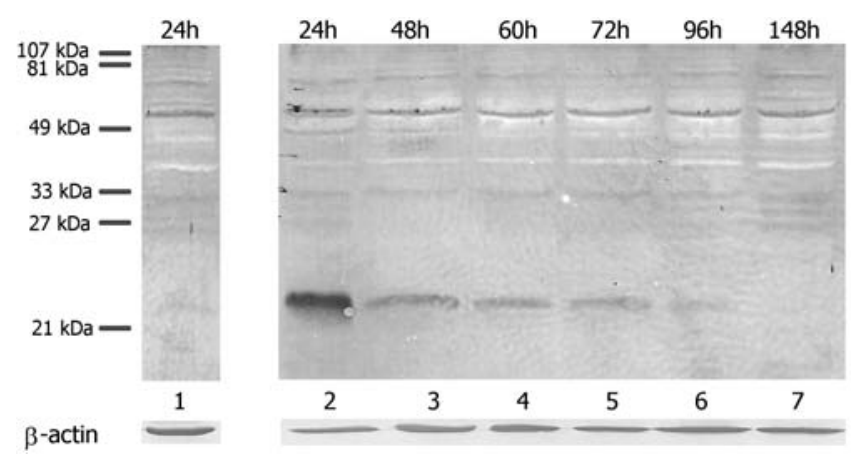

Figure 2. SDS-PAGE and an immunoblot of chondrocyte extracts. Lane 1, chondrocytes cultured for $24 \mathrm{~h}$ and detached by trypsin. Lane 2-7, chondrocytes cultured in monolayer for 24, 48, 60, 72, 96 and $148 \mathrm{~h}$ and detached by collagenase.

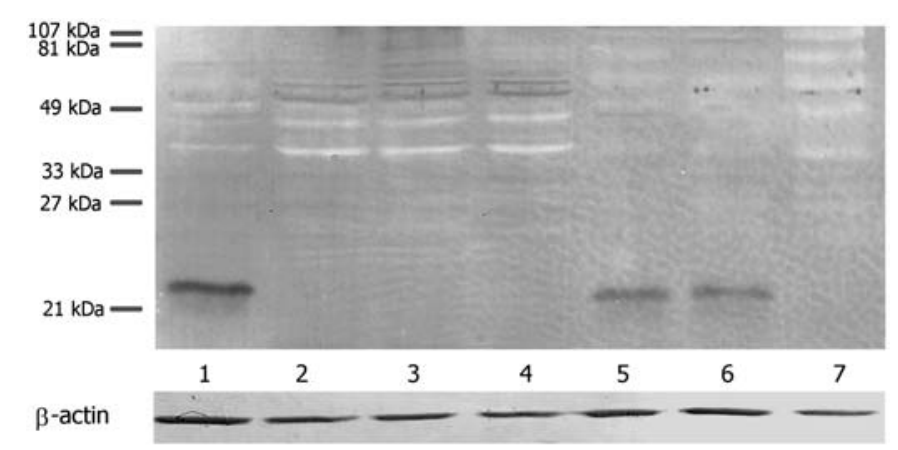

Figure 3. SDS-PAGE and an immunoblot of chondrocyte extracts. Lane 1-4, chondrocytes cultured in monolayer for $1,7,14$ or 21 days. Lane 5 , freshly isolated chondrocytes and lane 6 and 7, chondrocytes after 7 or 14 days of monolayer culture re-cultured in Matrigel for 7 days.

electrophoresis (SDS-PAGE; 12\% acrylamide) (14). Separated proteins were transferred onto PVDF membrane by semi-dry blotting at $25 \mathrm{~V}$ for 30 min using Trans-Blot SD apparatus (Bio-Rad Laboratories, Hercules, CA, USA). Serum from rat chondrocyte-sensitized rabbits diluted 1:120 served as the first antibody (10). Incubation lasted for $1 \mathrm{~h}$. Biotinylated $\mathrm{F}\left(\mathrm{ab}^{\prime}\right)_{2}$ fragments of swine anti-rabbit immunoglobulins (Dako A/S, Glostrup, Denmark) served as the second antibody. Actin used 


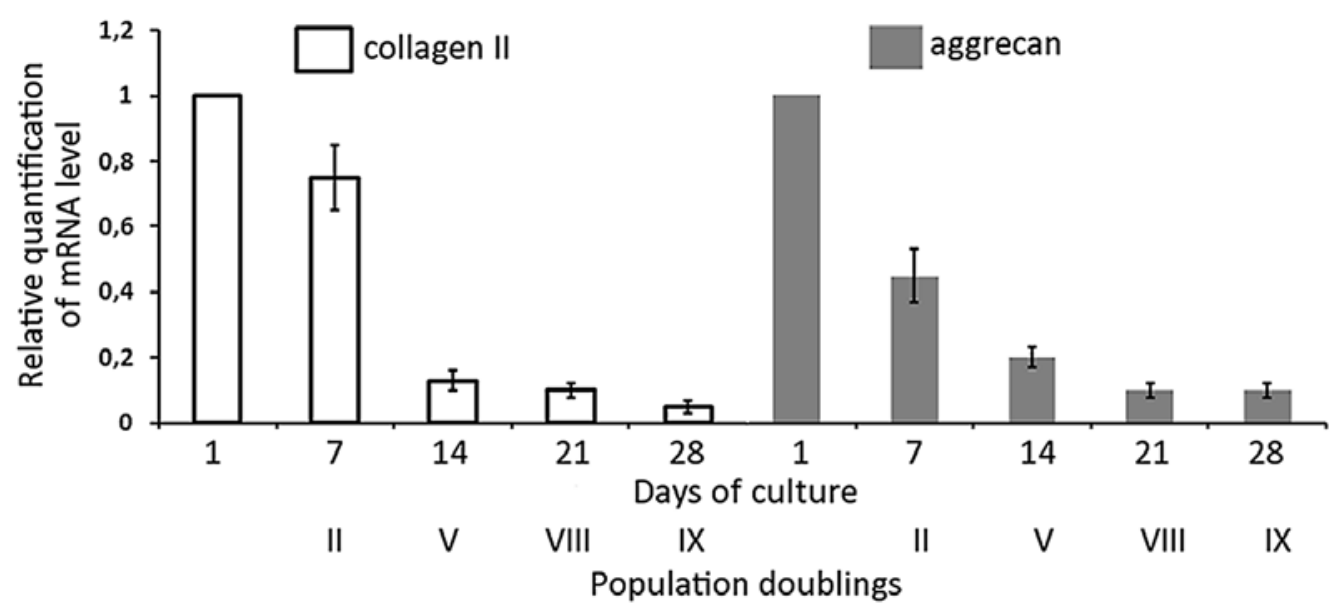

Figure 4. Relative quantification of mRNA level for collagen type II and aggrecan during monolayer culture of rat chondrocytes, measured by real-time PCR. The relative expression was related to the reference gene, GAPDH. Bars represent the mean values \pm SE from 3 different experiments. Analysis was conducted as a relative quantification study using the first day of culture gene expression as a calibrator (value $=1$ ). Chondrocytes during passages were detached with trypsin-EDTA.

as the internal control was detected with mouse monoclonal anti- $\beta$-actin antibody (Sigma) followed by biotinylated goat anti-mouse immunoglobulins (Dako). Antibody binding was demonstrated by an amplified alkaline phosphatase detection system (Bio-Rad). The relative molecular weight of the antigens was calculated using GelWorks 1D Intermediate software (Bio-Rad) in relation to the pre-stained SDS-PAGE (low range) standard (Bio-Rad).

Total RNA isolation. RNA was isolated using the NucleoSpin ${ }^{\circledR}$ RNA II Kit (Macherey-Nagel, Duren, Germany) according to the manufacturer's protocol. The quality of isolated RNA was determined by electrophoresis in $1 \%$ agarose denaturing gel containing $6 \%$ formaldehyde and buffered with MOPS (Sigma). After electrophoresis, the gel was scanned by the GDS9000 digital imaging system using GRAB-IT.2.0 software (UVP, Cambridge, UK). Densitometric analysis of the bands was performed with GelWorks software (UVP).

Reverse transcription. Reverse transcription was performed using a High-Capacity cDNA Reverse Transcription kit (Applied Biosystems, Cheshire, UK) according to the manufacturer's protocol in an Eppendorf Mastercycler Gradient. cDNA samples were stored at $-20^{\circ} \mathrm{C}$.

Real-time PCR. Real-time PCR was performed using an ABI PRISM 7500 system (Applied Biosystems) in 96-well optical plates. Each sample was tripled and supplied with endogenous control (Rat GAPDH Endogenous Control VIC ${ }^{\circledR} /$ MGB Probe). For gene expression, TaqMan Expression Assays were used: Rn00563954_m1 for collagen II, Rn00573424_ml for aggrecan, Rn01526721_m1 for collagen I and Rn01493763_m1 for versican. All probes were stained with FAM (Applied Biosystems). Reactions were run in a $25-\mu 1$ volume with TaqMan Universal Master Mix, the appropriate primer set, an MGB probe and $50 \mathrm{ng}$ cDNA template. Universal thermal conditions of $10 \mathrm{~min}$ at $95^{\circ} \mathrm{C}$ and 40 cycles of $15 \mathrm{sec}$ at $95^{\circ} \mathrm{C}$ and $1 \mathrm{~min}$ at $60^{\circ} \mathrm{C}$ were used. Data analysis was performed using Sequence Detection Software version 1.2 (Applied Biosystems).

\section{Results}

After $24 \mathrm{~h}$ of 2-D culture, freshly isolated chondrocytes assumed a multiangular shape. After 7 days, corresponding to 2 population doublings, the cells became more elongated. After 21 days (8 population doublings), they acquired a fibroblastlike phenotype. Proliferation of chondrocytes in 3 independent experiments ceased after 9 population doublings. Freshly isolated chondrocytes introduced into Matrigel (3-D culture) remained spherical after 1 and 7 days. Chondrocytes cultured for 7 days in monolayer and transferred into Matrigel for another 7 days had a similar appearance as freshly isolated chondrocytes cultured in Matrigel. Chondrocytes cultured for 14 days in monolayer and then re-cultured in Matrigel formed clumps. However, cells at the periphery of the clumps remained spherical.

As reported previously, CAA has an $\mathrm{M}_{\mathrm{r}}$ of $\sim 74 \mathrm{kDa}$ in the non-reduced and $\sim 23 \mathrm{kDa}$ in the reduced state (Fig. 1) (10). CAA was not detected in chondrocytes extracts cultured in monolayer for $24 \mathrm{~h}$ and detached from the vessel with trypsinEDTA (Fig. 2). In contrast, cells detached with collagenase retained CAA. In 2-D culture, the amount of CAA decreased with time, but was still detectable in 96-h cultures (Fig. 2). Freshly isolated chondrocytes or those cultured for 7 days in monolayer detached by collagenase and introduced into Matrigel for 1 week expressed CAA. Chondrocytes cultured for 14 days in monolayer and re-cultured in Matrigel for 7 days did not re-express CAA (Fig. 3).

In comparison with cells cultured for $24 \mathrm{~h}$, the expression of collagen type II and of aggrecan in chondrocytes cultured for 7 days in monolayer and detached with trypsin-EDTA decreased and dropped to a very low level after 14 days (Fig. 4). Expression of collagen type I and versican markedly increased after 7 days and remained at high levels after up to 28 days of 2-D culture (Fig. 5).

In chondrocytes cultured in monolayer and detached by collagenase, expression of collagen type II and aggrecan was decreased after 7 days and remained low until the end of the experiment. Freshly isolated chondrocytes and those cultured for 7 days in monolayer exhibited markedly increased expres- 


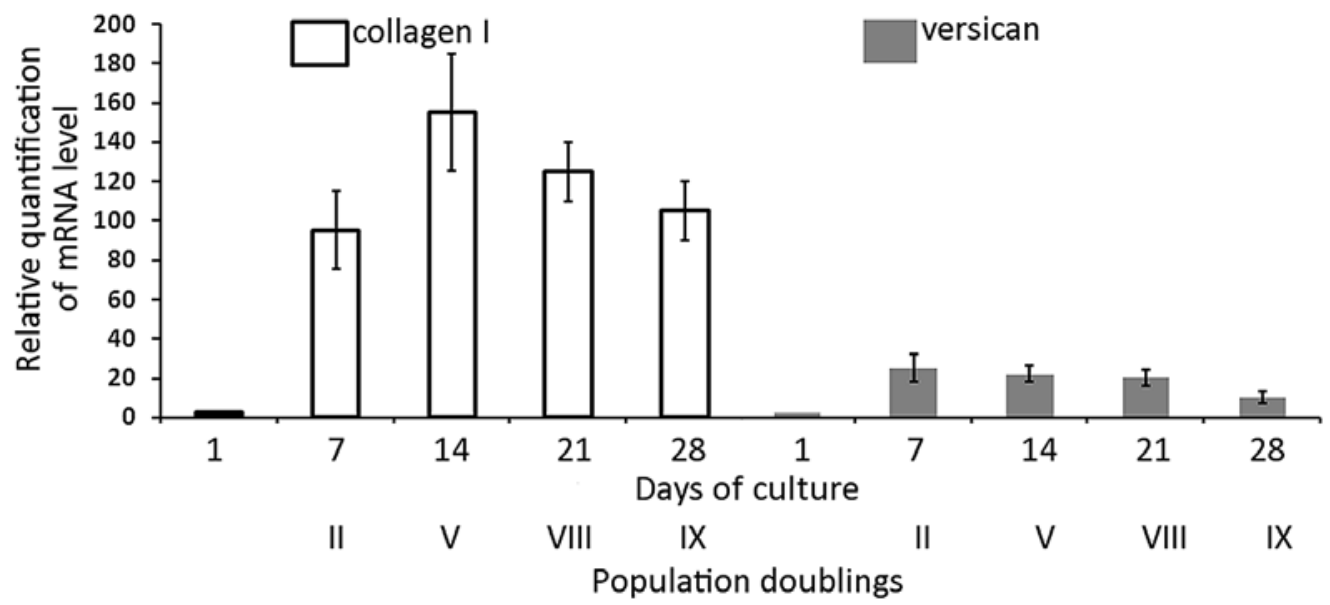

Figure 5. Relative quantification of mRNA level for collagen type I and versican during monolayer culture of rat chondrocytes, measured by real-time PCR. The relative expression was related to the reference gene, GAPDH. Bars represent the mean values \pm SE from 3 different experiments. Analysis was conducted as a relative quantification study using the first day of culture gene expression as a calibrator (value $=1)$. Chondrocytes during passages were detached with trypsin-EDTA.

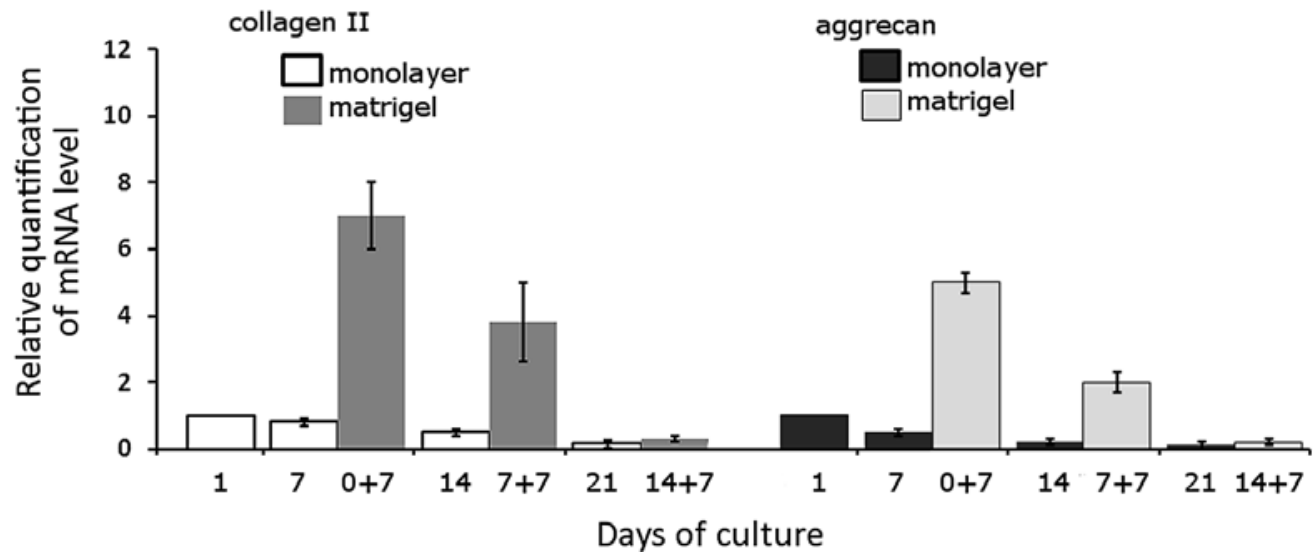

Figure 6. Relative quantification of mRNA level for collagen type II and aggrecan during monolayer (2-D) or Matrigel (3-D) culture of rat chondro-cytes, measured by real-time PCR. Chondrocytes during passages were detached with collagenase and seeded into Matrigel directly after isolation and after 7 or 14 days of culture in a monolayer. Chondrocytes in Matrigel were cultured for 1 week. The relative mRNA levels were related to the reference gene, GAPDH. Bars represent the mean values \pm SE from 3 different experiments. Analysis was conducted as a relative quantification study using the first day of culture gene expression as a calibrator (value $=1$ ).

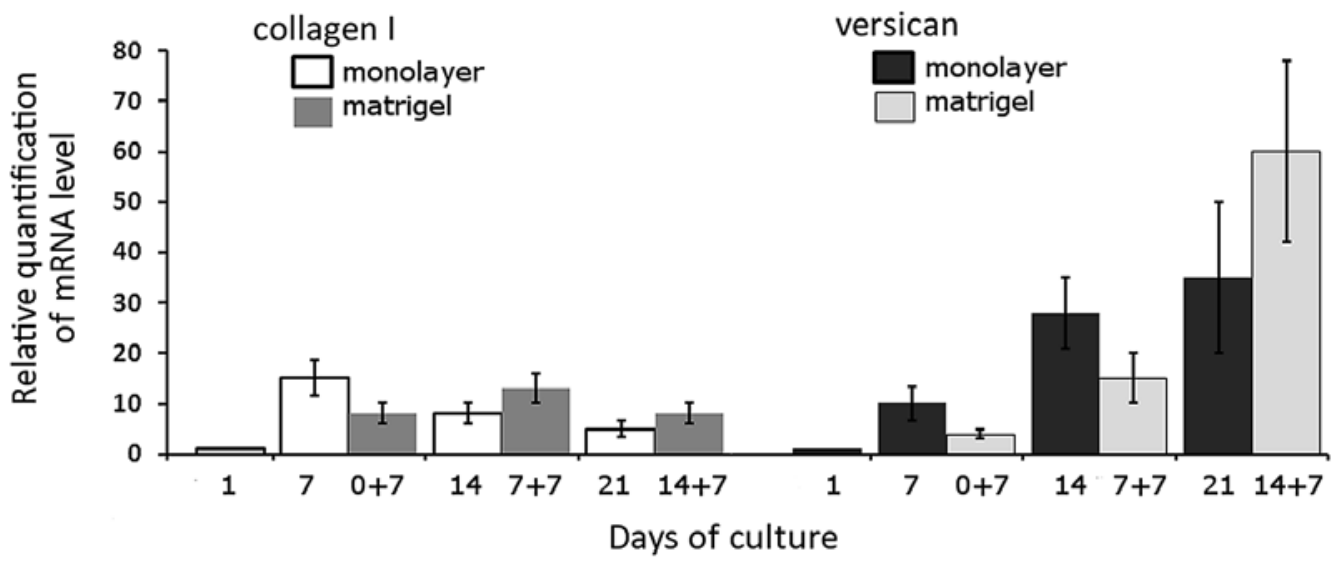

Figure 7. Relative quantification of the mRNA level for collagen type I and versican during monolayer (2-D) or Matrigel (3-D) culture of rat chondrocytes, measured by real-time PCR. Chondrocytes during passages were detached with collagenase and seeded into Matrigel directly after isolation and after 7 or 14 days of culture in a monolayer. Chondrocytes in Matrigel were cultured for 1 week. The relative mRNA levels were related to the reference gene, GAPDH. Bars represent the mean values \pm SE from 3 different experiments. Analysis was conducted as a relative quantification study using first day of culture gene expression as a calibrator (value $=1$ ). 
sion of collagen type II and aggrecan when seeded into Matrigel for 1 week. Chondrocytes cultured for 14 days in monolayer did not recover collagen type II or aggrecan expression in Matrigel (Fig. 6).

Expression of collagen type I increased after chondrocytes were cultured in monolayer for 7 days, then slightly declined over the subsequent two weeks. The culture of chondrocytes in Matrigel had no clear effect on collagen type I expression. Expression of versican steadily rose in monolayer cultures lasting 7-21-days. In chondrocytes incorporated into Matrigel immediately after isolation and after 7 days of monolayer culture, the expression of versican was less pronounced than in chondrocytes from monolayers. Versican expression was further augmented in chondrocytes cultured for 14 days in monolayer and re-cultured in Matrigel (Fig. 7).

\section{Discussion}

In this study, we observed that CAA gradually disappeared in chondrocytes during monolayer culture, but could be detected after the first passage on day 4 of culture when cells were detached by collagenase. In contrast, trypsin-EDTA treatment removed CAA from chondrocytes cultured in monolayer for $24 \mathrm{~h}$. Similarly, trypsin and pronase used for tissue dissociation were found to absorb to the cell surface, retaining activity even after triplicate washing and interfering with the formation of the glycoprotein cell coat (15). Moreover, proteolytic enzymes affected cell morphology (16). Chondrocyte gene expression levels of matrix components were also markedly influenced by the collagenase-based procedures used for cartilage digestion (17), and numerous chondrocyte surface markers were differentially affected by harvesting with trypsin/EDTA, pronase or collagenase (7).

It has previously been suggested that chondrogenic expression of cartilage cells is influenced by changes in cell shape. Cell rounding has been found to promote chondrogenesis while slowing down proliferation, while cell flattening stimulated proliferation but interfered with the expression of the chondrocyte phenotype (18). However, further studies have suggested that no conclusion can be drawn from the morphological appearance of cultured chondrocytes regarding the type of collagen the cell is producing, since rounded cells sometimes express collagen type I and flattened cells, collagen type II (19). These findings are further supported by a study which observed that human adult articular chondrocytes cultured as monolayers displayed fibroblastoid morphology and grew faster than those cultured as suspensions over agarose, which adopted a rounded shape with little or no DNA synthesis. However, under both culture conditions and similarly to intact human articular cartilage, chondrocytes expressed not only markers characteristic of cartilage, but also type I collagen. Thus, cell shape was not correlated with the expression of the chondrocyte phenotype, and the loss of the chondrocyte phenotype appeared to result from the loss of one or more cartilage-specific molecules, rather than from the expression of non-cartilage-specific molecules (20).

In our study, the mRNA levels for collagen type I and II, aggrecan and versican in rat chondrocytes cultured in monolayer and detached by trypsin-EDTA were high during the first week of culture when cell proliferation was low.
However, during the second and third weeks of culture, when cell proliferation accelerated, only the expression of collagen type I had a tendency to increase, while the expression of versican slightly and of collagen type II and aggrecan markedly decreased (Figs. 4 and 5). In human articular chondrocytes cultivated in monolayer for 56 days (with three passages and detachment of cells by trypsin-EDTA), mRNA for collagen type I was not expressed at the beginning of culture and increased 100-fold during the culture period. Collagen type II and IX expression decreased $>100$-fold. Aggrecan expression was down-regulated 100-fold, whereas versican expression increased 10 times (21). Thus, in both rat and human chondrocytes cultured in monolayer, the increase in collagen type I expression was more prominent than that of versican.

It is worth noting that, in all these studies, chondrocytes were isolated by collagenase and detached during culture passage with trypsin-EDTA. Expression of collagen type II and aggrecan in monolayer culture of chondrocytes detached by collagenase was similar to that observed after trypsinEDTA detachment. On the other hand, in the present study, expression of collagen type I increased during the first week (Figs. 6 and 7) and remained at this level throughout further cultivation, while expression of versican rose steadily over the whole cultivation period. Thus, the technique of chondrocyte detachment during passage may influence the expression of particular matrix molecules.

Benya and Schaffer (5) noted that the phenotypic changes of chondrocytes may be reversed when flattened rabbit chondrocytes from monolayer culture are transferred into agarose gel. In such cases, cells were observed to assume a spherical shape, and regained the characteristics of cartilage collagen and matrix proteoglycan expression, with the complete disappearance of collagen type I. Similar results were obtained with human epiphyseal chondrocytes which, after monolayer culture for 1-5 passages, produced type I procollagen, fibronectin and small non-cartilaginous proteoglycans. After transfer to agarose for 1 or 2 weeks, chondrocytes resumed production of collagen type II and cartilage-specific proteoglycans. Nevertheless, a few cells in monolayer culture immunostained for collagen type II, and some grown in agarose contained collagen type I. The number of such cells increased when the culture in agarose was prolonged to 3 weeks (22). Redifferentiation of dedifferentiated human chondrocytes was also observed in high density cultures $(23,24)$. In another 3 -D culture system, human embryonic chondrocytes expanded in monolayer culture for several days without passages and then transferred into alginate beads also reverted to a chondrocytic phenotype (6).

Matrigel used in the present study as a 3-D system for chondrocyte cultivation differs from other 3-D supporters because it contains several growth factors and connective tissue components. Mesenchymal stem cells from the bone marrow of osteoarthritis patients cultured for 7 days in Matrigel in chondrogenic medium expressed collagen IIA and aggrecan mRNA (11). In our study, chondrocytes expanded in monolayer culture for 1 week and re-cultured in Matrigel for 1 week regained expression of CAA as well as collagen type II and aggrecan. After 2 weeks of monolayer culture, CAA was undetectable in chondrocytes re-cultured in Matrigel, 
and collagen type II as well as aggrecan were expressed at a very low level in comparison with a previous group (Fig. 6). Expression of collagen type I in chondrocytes re-cultured in Matrigel after 1 or 2 weeks in monolayer remained at a similar level, while expression of versican considerably increased after a 2-week-long expansion in monolayer. Thus, it appears that culture in Matrigel allows for increased expression of cartilage-specific molecules without suppression of collagen type I and versican. In this respect, it differs from the agarose or alginate bead systems. Moreover, the return of CAA expression is correlated with collagen type II and aggrecan mRNA levels. This suggests that CAA may be used as a marker of chondrocyte differentiation.

It is also noteworthy that native Matrigel contains several growth factors and basement membrane components. The addition of growth factors stimulated the expansion of chondrocytes in monolayer cultures and redifferentiation in dense 2 -D or 3 -D cultures $(23,25,26)$. The growth factors present in Matrigel, i.e., TGF- $\beta$, PDGF and IGF-1, were previously found to stimulate the re-expression of the chondrocytic phenotype after the transfer of cells from 2-D to 3-D culture (26-28). This finding is in close agreement with the observation that chondrocytes express TGF- 3 , PDGF and IGF-1 receptors $(7,29)$. Furthermore, laminin present in Matrigel was observed to serve as a substrate for the anchorage of chondrocytes (30), which can be explained by the presence on chondrocytes of laminin-binding integrin receptors (31-33).

On the other hand, rat chondrocytes remaining in monolayer cultures for 14 days lost the ability to redifferentiate in 3 -D cultures. Redifferentiation occurred much faster in rat than in rabbit $(5)$ or human chondrocytes $(6,22)$, and probably mirrors interspecies differences as well as conditions of culture. It was previously observed by Coon (34) that the cartilage phenotype is more stable when chondrocytes are grown as clones. The addition of a small number of noncartilage cells to chick embryo clonal chondrocyte strains resulted in the rapid loss of cartilage phenotype (35). These previous observations have achieved a new meaning due to the recent discovery that human articular cartilage contains cells with stem cell properties capable of acquiring features characteristic of chondrogenic, osteogenic or adipogenic differentiation $(26,36)$. It has also been demonstrated that the cells of chondrogenic lineage stimulated by growth factors (TGF-B1, FGF 2 and PDGF type BB) displayed marked upregulation of type II collagen and glycosaminoglycan/DNA content (26). Moreover, human chondrocytes with high chondrogenic capacity were found to express markers associated with cell-cell or cell-matrix interactions, while a low capacity for matrix production was associated with higher expression of IGF-1 and catabolic genes, e.g., matrix metalloproteinases (37). In relation to the findings presented above, it is also important to note that articular cartilage chondrocytes differ in response to IGF-1 depending on the zone from which they were isolated (38).

In view of early observations suggesting the inhibitory influence of non-cartilage cells on cartilage matrix molecule expression (35), in the present study we attempted to eliminate the possibility of contamination of cartilage by fibroblasts. Only cartilage fragments which could be mechanically cleared of adhering tissues were isolated. Additionally, they were exposed to trypsin (without EDTA) to remove cells which could be missed during dissection. This procedure did not, however, eliminate cells of non-chondrocytic lineage present within the cartilage. The presence of such cells among freshly isolated chondrocytes is suggested by the appearance of collagen type I and versican mRNA in Matrigel cultures. Thus, it seems plausible that during prolonged expansion of chondrocytes in monolayer culture, cells with chondrogenic potential become suppressed and are unable to redifferentiate after transfer into 3-D culture.

\section{Acknowledgements}

This study was supported by the State Committee for Scientific Research (KBN) grant 2 PO5A 11228.

\section{References}

1. Nameroff $\mathrm{M}$ and Holtzer H: The loss of phenotypic traits by differentiated cells. IV. Changes in polysaccharides produced by dividing chondrocytes. Dev Biol 16: 250-281, 1967.

2. Chacko S, Abbott J, Holtzer S and Holtzer H: The loss of phenotypic traits by differentiated cells. VI. Behavior of the progeny of a single chondrocyte. J Exp Med 130: 417-442, 1969.

3. Mayne R, Vail MS, Maine PM and Miller EJ: Changes in type of collagen synthesis as clones of chick chondrocyte grow and eventually lose division capacity. Proc Natl Acad Sci USA 73: 1674-1678, 1976.

4. Schlitz JR, Mayne R and Holtzer H: The synthesis of collagen and glycosaminoglycans by dedifferentiated chondrocytes in culture. Differentiation 1: 97-108, 1973.

5. Benya PD and Shaffer JD: Dedifferentiated chondrocytes reexpress the differentiated collagen phenotype when cultured in agarose gels. Cell 30: 215-224, 1982.

6. Bonaventure J, Kadhom N, Cohen-Solal L, Ng KH, Bourguignon J and Lasselin C: Reexpression of cartilage-specific genes by dedifferentiated human articular chondrocytes cultured in alginate beads. Exp Cell Res 212: 97-104, 1994.

7. Diaz-Romero J, Gaillard JP, Grogan SP, Nesic D, Trub T and Mainil-Varlet P: Immunophenotypic analysis of human articular chondrocytes: changes in surface markers associated with cell expansion in monolayer culture. J Cell Physiol 202: 731-742, 2005.

8. Goessler UR, Bieback K, Bugert P, Naim R, Schafer C, Sadick H, Hormann K and Riedel F: Human chondrocytes differentially express matrix modulators during in vitro expansion for tissue engineering. Int J Mol Med 16: 509-515, 2005.

9. Glant T and Mikecz K: Antigenic profiles of human, bovine and canine articular chondrocytes. Cell Tissue Res 244: 359-369, 1986.

10. Osiecka-Iwan A, Hyc A, Jozwiak J, Komar A, Niderla J and Moskalewski S: Transplants of rat chondrocytes evoke strong humoral response against chondrocyte-associated antigen in rabbits. Cell Transplant 12: 389-398, 2003.

11. Warnawin E, Burakowski T, Gajewski M, Radzikowska A, Kornatka A, Michalak C, Maldyk P, Maslinski S and Maslinski W: Preservation of chondrogenic potential of mesenchymal stem cells isolated from osteoarthritic patients during proliferation in response to platelet-derived growth factor (PDGF). Centr Eur J Immunol 30: 26-31, 2005.

12. Osiecka-Iwan A, Hyc A and Moskalewski S: Immunosuppression and rejection of cartilage formed by allogeneic chondrocytes in rats. Cell Transplant 8: 627-636, 1999.

13. Schägger $\mathrm{H}$ and von Jagow G: Blue native electrophoresis for isolation of membrane protein complexes in enzymatically active form. Anal Biochem 199: 223-231, 1991.

14. Laemmli UK: Cleavage of structural proteins during the assembly of the head of bacteriophage T4. Nature 227: 680-685, 1970.

15. Poste G: Tissue dissociation with proteolytic enzymes. Adsorption and activity of enzymes at the cell surface. Exp Cell Res 65: 359-367, 1971

16. Thyberg J and Moskalewski S: Influence of proteolytic enzymes and calcium-binding agents on nuclear and cell surface topography. Cell Tissue Res 237: 587-593, 1984. 
17. Hayman DM, Blumberg TJ, Scott CC and Athanasiou KA: The effects of isolation on chondrocyte gene expression. Tissue Eng 12: 2573-2580, 2006.

18. Glowacki J, Trepman E and Folkman J: Cell shape and phenotypic expression in chondrocytes. Proc Soc Exp Biol Med 172: 93-98, 1983.

19. Von der Mark K, Gauss V, von der Mark H and Müller P: Relationship between cell shape and the type of collagen synthesised as chondrocytes lose their cartilage phenotype in culture. Nature 267: 531-532, 1977.

20. Kolettas E, Buluwela L, Bayliss MT and Muir HI: Expression of cartilage-specific molecules is retained on long-term culture of human articular chondrocytes. J Cell Sci 108: 1991-1999, 1995.

21. Marlovits S, Hombauer M, Tamandl D, Vèscei V and Schlegel W: Quantitative analysis of gene expression in human articular chondrocytes in monolayer culture. Int J Mol Med 13: 281-287, 2004.

22. Aulthouse AL, Beck M, Griffey E, Sandford J, Arden K, Machado MA and Horton WA: Expression of the human chondrocyte phenotype in vitro. In Vitro Cell Dev Biol 25: 659-668, 1989.

23. Bradham DM and Horton WE Jr: In vivo cartilage formation from growth factor modulated articular chondrocytes. Clin Orthop Relat Res 352: 239-249, 1998.

24. Schulze-Tanzil G, de Souza P, Villegas Castrejon H, John T, Merker H-J, Scheid A and Shakibaci M: Redifferentiation of dedifferentiated human chondrocytes in high density cultures. Cell Tissue Res 308: 371-379, 2002.

25. Jakob M, Démarteau O, Schäfer D, Hintermann B, Dick W, Heberer $M$ and Martin I: Specific growth factors during the expansion and redifferentiation of adult human articular chondrocytes enhance chondrogenesis and cartilaginous tissue formation in vitro. J Cell Biochem 81: 368-377, 2001.

26. Barbero A, Ploegert S, Heberer M and Martin I: Plasticity of clonal populations of dedifferentiated adult human articular chondrocytes. Arthritis Rheum 48: 1315-1325, 2003.

27. Yaeger PC, Masi TI, de Ortiz JL, Binette F, Tubo R and McPherson JM: Synergistic action of transforming growth factorbeta and insulin-like growth factor-1 induces expression of type II collagen and aggrecan genes in adult human articular chondrocytes. Exp Cell Res 237: 318-325, 1997.
28. Goldberg AJ, Lee DA, Bader DL and Bentley G: Autologous chondrocyte implantation. Culture in a TGF-beta-containing medium enhances the re-expression of a chondrocytic phenotype in passaged human chondrocytes in pellet culture. J Bone Joint Surg 87: 128-134, 2005.

29. Goessler UR, Bugert P, Bieback K, Sadick H, Baisch A, Hormann $\mathrm{K}$ and Riedel $\mathrm{F}$ : In vitro analysis of differential expression of collagens, integrins, and growth factors in cultured human chondrocytes. Otolaryngol Head Neck Surg 134: 510-515, 2006.

30. Bulic K: Articular chondrocytes interact with basement membrane Matrigel through laminin active binding sites. Acta Med Croatica 50: 69-74, 1996.

31. Albelda SM and Buck CA: Integrins and other cell adhesion molecules. FASEB J 4: 2868-2880, 1990.

32. Hirsch MS, Lunsford LE, Trinkaus-Randall V and Svoboda KKH: Chondrocyte survival and differentiation in situ are integrin mediated. Dev Dyn 210: 249-263, 1997.

33. Hynes RO: Integrins: versatility, modulation, and signaling in cell adhesion. Cell 69: 11-25, 1992.

34. Coon HG: Clonal stability and phenotypic expression of chick cartilage cells in vitro. Proc Natl Acad Sci USA 55: 66-73, 1966.

35. Bryan J: Studies on clonal cartilage strains. Effect of contaminating non-cartilage cells. Exp Cell Res 52: 319-326, 1968.

36. Thornemo M, Tallheden T, Sjorgen Jansson E, Larsson A, Lovstedt K, Nannmark U, Brittberg M and Lindahl A: Clonal populations of chondrocytes with progenitor properties identified within human articular cartilage. Cells Tissues Organs 180: $141-150,2005$.

37. Grogan SP, Barbero A, Diaz-Romero J, Cleton-Jansen AM, Soeder S, Whiteside R, Hogendoorn PC, Farhadi J, Aigner T, Martin I and Mainil-Varlet P: Identification of markers to characterize and sort human articular chondrocytes with enhanced in vitro chondrogenic capacity. Arthritis Rheum 56: 586-595, 2007.

38. Eleswarapu SV, Leipzig ND and Athanasion KA: Gene expression of single articular chondrocytes. Cell Tissue Res 327: 43-54, 2007. 